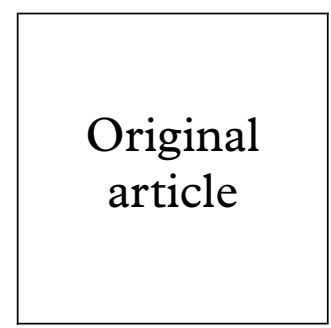

\author{
Richard M Shattock, Craig Patrizio, Peter Simmonds, Sheena Sutherland
}

\section{Detection of Chlamydia trachomatis in genital swabs: comparison of commercial and in house amplification methods with culture}

\begin{abstract}
Aims: To evaluate the sensitivity of the Roche Cobas, Roche Amplicor plate kit, ligase chain reaction (LCR), and an in house polymerase chain reaction (PCR) by titration of purified elementary bodies (EB) and also to test 245 urethral and endocervical specimens for Chlamydia trachomatis by the four assays as well as conventional culture.

Study design: EB titrations were run in duplicate in each commercial assay and six times in the in house PCR. Clinical samples were aliquoted and tested by each assay and were considered positive if $C$ trachomatis was detected by two or more separate tests or if the sample was either culture or immunofluorescence positive. Major outer membrane protein (MOMP) specific primers were used as a confirmatory assay for the in house PCR.

Results: The in house PCR, Roche Cobas Amplicor, LCR, and Amplicor plate kit gave detection limits of approximately 1, 1-2, 2, and 2-4 EBs respectively. By the criteria described above for definition of a $C$ trachomatis positive result in clinical samples we identified 23 true positives among the 245 clinical specimens. The in house PCR detected all 23 giving a sensitivity of $100 \%$ and a specificity of $98 \%$. The Roche Cobas Amplicor, Roche Amplicor plate kit, and LCR detected 21,19 , and 19 of these respectively giving sensitivities of $87.5 \%, 82 \%$, and $82 \%$ respectively and specificities of $99.5 \%, 99 \%$, and $100 \%$ respectively. The culture gave a sensitivity of $78 \%$ and specificity of $100 \%$.

Conclusion: All four amplification assays had a greater sensitivity than the culture used routinely in this laboratory. The in house plasmid PCR had the greatest sensitivity and when combined with confirmation by immunofluorescence detected the greatest number of positives. This increased sensitivity is likely to have been achieved by the use of a DNA purification step and of nested primers in the amplification stage and their combined use in routine diagnostic assays for chlamydia might increase the frequency of $C$ trachomatis detections. However, this assay is much less user friendly than the two semiautomated commercial assays investigated in this study. (Sex Transm Inf 1998;74:289-293)
\end{abstract}

Keywords: genital swabs; Chlamydia trachomatis; amplification methods

\section{Introduction}

Chlamydia trachomatis serovars $\mathrm{D}-\mathrm{K}$ are the commonest cause of non-gonococcal urethritis in males and mucopurulent cervicitis and urethritis in females. Many patients are asymptomatic and if untreated they will be a risk both to their partners and to themselves. For example ascending infection can occur, and in females can result in pelvic inflammatory disease (PID) causing ectopic pregnancies and infertility. It is therefore desirable to identify chlamydial infections in infected individuals and administer appropriate treatment before further complications arise.

Laboratory diagnosis of $C$ trachomatis was originally based on the culture of urethral and endocervical swabs. ${ }^{1}$ More recently, attempts have been made to develop more rapid methods, such as the detection of chlamydial antigen by immunological assays. Among these, direct fluorescent antibody assays (DFA) and enzyme linked immunosorbent assays (ELISA) using monoclonal antibodies directed against chlamydial antigens have become widely used. These show sensitivities similar to that of culture, although precise comparisons are influenced by the methods of sample collection, transport, and storage. ${ }^{23}$

Alternatives to immunological detection are methods based upon the amplification of bacterial nucleic acid sequences. ${ }^{4}$ Two techniques developed for the detection of $C$ trachomatis are the ligase chain reaction (LCR, Abbott) ${ }^{56}$ and Roche Cobas Amplicor. Both methods amplify sequences from a cryptic plasmid present within bacterial cells at copy numbers of $7-10 .{ }^{7}$ These methods may therefore be more sensitive than amplification of single gene sequences from the bacterial genome such as those coding for the major outer membrane protein (MOMP). The Roche Cobas Amplicor is an automated system of the conventional Roche Amplicor polymerase chain reaction (PCR) plate kit. Like the LCR, the Roche Cobas has a manual sample processing but allows automated amplification and detection as well as result reporting.

Our study involved the comparison of Roche Cobas Amplicor, Roche Amplicor plate kit, Abbott LCR, in house PCR, and culture on swabs collected routinely over 2 weeks from 245 patients attending a single genitourinary medicine clinic (GUM) (221) and a single local general practice (24). 


\section{Materials and methods}

STUDY POPULATION

The specimens used in the study were consecutive swabs sent in sucrose phosphate (2SP) transport medium for routine culture (251) over 10 working days with the exception of six samples. Three of the excluded samples were conjunctival swabs, one was a rectal swab, and two had insufficient 2SP for all the tests to be performed. The remaining 245 were collected from 120 males and 125 females. Urethral swabs numbered 123 (120 male, three female), endocervical swabs 121 , and one female swab was site unknown. The GUM patients comprised 138 patients attending for the first time, 66 previous attenders with a new episode of illness, and 20 previous attenders returning for follow up of a recent episode. None of the general practitioner patients was on follow up visits.

COLLECTION OF SAMPLES

To avoid the swab sample variation that can occur when multiple swabs are taken, only one swab was submitted for each patient. The swab sent for chlamydia culture was the second swab taken, the first swab being sent for bacteriology. The volume of 2SP was not increased so that our routine culture sensitivity would not be compromised and to ensure that our service to the patients remained as normal. The swabs were received in approximately $1.2 \mathrm{ml}$ of $2 \mathrm{SP}$ transport medium. Specimens were vortexed for 1 minute, $500 \mu \mathrm{l}$ of the specimen was cultured, and four aliquots of $100 \mu \mathrm{l}$ were used for each of the other tests. The remaining specimen (approximately $300 \mu \mathrm{l}$ ) was stored for immunofluorescence (IF) if required.

The LCR (Abbott) required resuspension of the sample in manufacturer's diluent as 2SP is unsuitable for this test. Aliquots tested by this method were prepared according to the manufacturer's instructions for testing urine samples and so were centrifuged at $15000 \mathrm{rpm}$ for 10 minutes, the supernatant discarded, and pellets stored at $-70^{\circ} \mathrm{C}$. Pellets were resuspended in $100 \mu \mathrm{l}$ of the manufacturer's urine resuspension buffer before testing.

\section{ELEMENTARY BODY TITRATIONS}

Elementary bodies (EBs) were purified and dilutions made in phosphate buffered saline (PBS). Dilutions containing 1000, 100, 10, 4, 2,1 , and $0.1 \mathrm{EBs}$ in $100 \mu \mathrm{l}$ were run in duplicate on each assay except the in house PCR which was repeated six times. The detection limit was calculated by taking the dilution factor into account during sample processing and assuming 10 plasmid copies per EB. All assays used $100 \mu \mathrm{l}$ volumes. DNA for the in house

Table 1 In house PCR primer sequences

\begin{tabular}{lll}
\hline Region & Primer & Sequence 5'-3' \\
\hline Plasmid & Outer sense & CTTTGCGCACAGACGATCTA \\
& Outer antisense & CAAATACTCTCCCATTTCTCC \\
& Inner sense & GCATCCAATCAATCAGATTTCC \\
Inner antisense & GTGTATTTTGCAACTCCTCC \\
OOMP & Outer sense & GAAAAAACTTRAARTCGG \\
& Outer antisense & CGNANGCTWATRGCRTCRCACCAAG \\
& Inner sense & TGCCTGTRGGGAAYCCWKCTGAWCCAAG \\
& Inner antisense & CAAGTNCNRCAAGGATCRCAAGGATC \\
\hline
\end{tabular}

Ambiguities: $\mathrm{N}=\mathrm{A}, \mathrm{T}, \mathrm{G}, \mathrm{C} ; \mathrm{W}=\mathrm{A} / \mathrm{T} ; \mathrm{R}=\mathrm{A} / \mathrm{G} ; \mathrm{K}=\mathrm{G} / \mathrm{T} ; \mathrm{Y}=\mathrm{C} / \mathrm{T}$.
PCR was eluted in $50 \mu$ of which $5 \mu$ was used in the PCR (1/10). The Roche Cobas and Roche Amplicor plate kit used $50 \mu$ of prepared sample from a total volume of $400 \mu \mathrm{l}(1 / 8)$. The LCx used all of the $100 \mu \mathrm{l}$ in the amplification

CHLAMYDIA CULTURE

Volumes of $500 \mu \mathrm{l}$ of each sample were inoculated with centrifugation at $37^{\circ} \mathrm{C}$ for 1 hour onto monolayers of McCoy cells in the presence of cycloheximide and incubated for 72 hours at $37^{\circ} \mathrm{C}$. Cells were stained with iodine and inspected microscopically for the presence of glycogen containing inclusions. ${ }^{89}$

IMMUNOFLUORESCENCE

Slides were prepared by cytospin method using $150 \mu \mathrm{l}$ of sample and fixed in methanol. PathoDX MOMP specific antibody was used for EB detection.

IN HOUSE PCR

DNA was extracted using the Qiamp viral RNA extraction kit. Nested primers (table 1) were used to amplify a $108 \mathrm{bp}$ fragment of the cryptic plasmid. The amplicon was detected by ethidium bromide staining after electrophoresis through a $2 \%$ agarose gel. A second assay using nested primers to amplify a $94 \mathrm{bp}$ fragment of the MOMP gene (table 1) was performed on all samples which gave a positive reaction with the plasmid assay.

ROCHE AMPLICOR PLATE KIT AND ROCHE COBAS AMPLICOR

All Roche assays were carried out according to the manufacturer's instructions. During the study Roche changed the sample preparation method for the plate kit so that the newer kit was used for the EB dilution series and the older kit for the 245 specimens.

ABBOTT LIGASE CHAIN REACTION

Pelleted samples, resuspended in LCx urine resuspension buffer, were tested according to the manufacturer's instructions.

DEFINITION OF A POSITIVE SAMPLE

All 245 clinical samples were tested by culture, in house PCR, and Roche Cobas Amplicor, Roche Amplicor plate kit, and Abbott LCR. Immunofluorescence was carried out on discrepant results as a confirmatory test and all plasmid positive samples were retested with MOMP primers. Samples were considered positive if (1) culture positive, (2) culture negative but positive by IF, or (3) positive in two or more of any other assays.

\section{Results}

By the criteria defined in the methods section a total of 23 of the $245(9.4 \%)$ clinical specimens were positive for $C$ trachomatis. Eleven (9\%) were male samples and $12(9.5 \%)$ were female samples.

\section{CLINICAL FEATURES}

Patients could be classified as asymptomatic, symptomatic possibly chlamydia related (SPC), and symptomatic but unrelated to chlamydia 
Table 2 (A) Presumed false positives

\begin{tabular}{|c|c|c|c|c|c|c|c|c|c|c|c|}
\hline$I D$ & Sex & Spec Type & Diag/Hist & $P P C R^{I}$ & $M P C R^{2}$ & Culture & $L C R$ & Cobas & Roche & $I F$ & Conclusion \\
\hline 1 & $\mathrm{M}^{3}$ & $\mathrm{U}^{4}$ & ASYM $^{5}$ & $(+/+)^{6}$ & - & - & - & - & - & - & Ind $^{15}$ \\
\hline 2 & $M$ & $\mathrm{U}$ & ASYM & $(+/-)$ & - & - & - & - & - & - & $\mathrm{IHP}^{7}$ false+ \\
\hline 3 & $\mathrm{~F}^{8}$ & $\mathrm{EC}^{9}$ & rash & $(+/-)$ & - & - & - & - & - & - & $"$ \\
\hline 4 & $\mathrm{~F}$ & EC & warts & $(+/-)$ & - & - & - & - & - & - & " \\
\hline 5 & $\mathrm{M}$ & $\mathrm{U}$ & TOC $^{10}$ & - & - & - & - & $(+/+)$ & - & - & Ind \\
\hline 6 & M & $\mathrm{U}$ & TOC & - & - & - & - & - & $(+/-)$ & - & Roche false+ \\
\hline 7 & $\mathrm{~F}$ & EC & V.DIS ${ }^{11}$ & - & - & - & - & - & $(+/-)$ & - & \\
\hline
\end{tabular}

(B) Presumed false negatives

\begin{tabular}{|c|c|c|c|c|c|c|c|c|c|c|c|}
\hline$I D$ & Sex & Spec Type & Diag/Hist & PPCR & $M P C R$ & Culture & $L C R$ & Cobas & Roche & $I F$ & Conclusion \\
\hline 8 & M & $\mathrm{U}$ & ASYM/Partner NSGI ${ }^{12}$ & $(+/+)$ & - & - & - & + & - & $2 \mathrm{EBs}$ & Low level+ \\
\hline 9 & M & U & TOC & $(+/+)$ & - & - & - & - & - & $3 \mathrm{EBs}$ & \\
\hline 10 & $\mathrm{~F}$ & $\mathrm{EC}$ & V.DIS & $(+/+)$ & - & + & - & - & - & $4 \mathrm{EBs}$ & ” \\
\hline 11 & M & $\mathrm{U}$ & NSGI & + & + & - & + & + & + & $6 \mathrm{EBs}$ & \\
\hline 12 & $\mathrm{~F}$ & $\mathrm{EC}$ & ASYM & $(+/+)$ & - & - & $(\mathrm{eq})^{13}$ & + & $(e q / e q)$ & - & Low levelt \\
\hline
\end{tabular}

Plasmid PCR; ${ }^{2}$ MOMP PCR $;{ }^{3}$ Male $;{ }^{4}$ Urethral $;{ }^{5}$ Asymptomatic; ${ }^{6}$ ( ) indicates results of screening and repeat assays; ${ }^{7}$ In house PCR; ${ }^{8}$ Female $;{ }^{9}$ Endocervical; ${ }^{10}$ Test of cure; ${ }^{11}$ Vaginal discharge; ${ }^{12}$ Partner with non-specific urethritis; ${ }^{13}$ Indicates an equivocal result; ${ }^{15}$ Indeterminate

(UC). There were 104 asymptomatic patients of whom 11 had returned for test of cure investigations and five were known contacts of chlamydia positive partners. Eighty three patients had symptoms unrelated to chlamydia and 58 had symptoms possibly related to chlamydia. The positivity rate in the three categories was (a) $10 \%$, (b) $2.4 \%$, and (c) $19 \%$. Of the 30 patients with NSGI, nine $(30 \%)$ were positive and two of the $27(7.4 \%)$ with vaginal discharge; one of these also had genital warts. Ten $(10 \%)$ of the asymptomatics were positive; two of these were tests of cure - one male, one female - and two were female contacts of known positives. The other asymptomatic patients were one male and five females. The remaining two positives were detected in two female patients with other STDs - one with herpes simplex infection the other with genital warts.

ASSAY RESULTS

Using the Roche Cobas Amplicor, 22 samples were positive, one of which was repeat Cobas positive but IF negative (table 2 , case 5 : indeterminate). The Roche plate kit detected 21 positives, 18 of which were culture positive while two of the remaining three samples were negative on repeat testing (table 2, cases 6 and 7). The other Roche positive was LCR positive

Table 3 Comparison of methods with confirmed results on sensitivity and specificity

\begin{tabular}{|c|c|c|c|c|}
\hline \multirow[b]{2}{*}{ Test and result } & \multicolumn{4}{|c|}{ Confirmed results } \\
\hline & Positive & Negative & Sensitivity (\%) & Specificity (\%) \\
\hline \multicolumn{5}{|c|}{ Plasmid PCR only* } \\
\hline positive & 23 & 4 & 100 & 98 \\
\hline negative & 0 & 218 & & \\
\hline \multicolumn{5}{|c|}{ Plasmid and MOMP PCR $†$} \\
\hline positive & 20 & 0 & 86 & 100 \\
\hline negative & 3 & 222 & & \\
\hline \multicolumn{5}{|l|}{ Roche Cobas } \\
\hline positive & 21 & 1 & 87.5 & 99.5 \\
\hline negative & 2 & 221 & & \\
\hline \multicolumn{5}{|l|}{ Roche plate kit } \\
\hline positive & 19 & 2 & 82 & 99 \\
\hline negative & 4 & 220 & & \\
\hline \multicolumn{5}{|l|}{ LCR } \\
\hline positive & 19 & 0 & 82 & 100 \\
\hline negative & 4 & 222 & & \\
\hline \multicolumn{5}{|l|}{ Culture } \\
\hline positive & 18 & 0 & 78 & 100 \\
\hline negative & 5 & 222 & & \\
\hline
\end{tabular}

*Sensitivities and specificities calculated if plasmid results only are taken.

†All positives with plasmid primers were retested using MOMP primers. A negative by MOMP primers was considered a false plasmid positive. IF results were not taken into account. and in house PCR positive but IF negative. There were also two Roche equivocals, one was negative on repeat testing and the second repeated equivocal (table 2 , case 12 ).

The LCR detected 19 positives and one equivocal which was IF negative but Cobas and in house PCR positive (table 2, case 12). There were no LCR false positives.

In house PCR gave a total of 27 positives using the plasmid primers. There were three presumed false positives which were negative by PCR using MOMP primers and negative upon repeat testing by the plasmid primers (table 2, cases 2, 3, and 4). One specimen was repeatedly positive using plasmid primers, MOMP assay negative and IF negative (table 2, case 1: indeterminate). By the criteria for positivity described above, these samples have been scored as false positives, although the latter sample may contain chlamydial DNA at a low level. Two specimens were repeatedly positive by plasmid primers, negative by MOMP primers, but positive by IF (cases 8 and 9). There was also one specimen positive by plasmid primers, culture positive, and IF positive with four EBs but was negative by all other methods (case 10). Table 3 compares sensitivity and specificity of resolved results in all the assays, with in house PCR results given before and after confirmation with the MOMP primers. Retesting with MOMP primers failed to detect three positives which were confirmed by IF. Confirmation of plasmid positive screens by MOMP primers would lower the sensitivity to $86 \%$ but would increase specificity to $100 \%$. Assuming all non-discrepant positives would have been IF positive, confirmation by IF would give a sensitivity of $95.6 \%$.

Table 4 Elementary body titrations for the three commercial assays and the in house PCR. 6/6 indicates six positive out of six tests performed

\begin{tabular}{llllllll}
\hline & \multicolumn{7}{c}{ Number of EBs present/100 $\mu l$} \\
\cline { 2 - 8 } Tests & 1000 & 100 & 10 & 4 & 2 & 1 & 0.1 \\
\hline $\begin{array}{l}\text { In house PCR } \\
\quad \text { (Plasmid) }\end{array}$ & $6 / 6$ & $6 / 6$ & $6 / 6$ & $6 / 6$ & $6 / 6$ & $6 / 6$ & $1 / 6$ \\
$\begin{array}{l}\text { In house PCR } \\
\quad(M O M P)\end{array}$ & $2 / 2$ & $2 / 2$ & $2 / 2$ & $0 / 2$ & $0 / 2$ & $0 / 2$ & $0 / 2$ \\
Roche Cobas & $2 / 2$ & $2 / 2$ & $2 / 2$ & $2 / 2$ & $2 / 2$ & $1 / 2$ & $0 / 2$ \\
Roche plate kit & $2 / 2$ & $2 / 2$ & $2 / 2$ & $2 / 2$ & $1 / 2$ & $0 / 2$ & $0 / 2$ \\
LCR & $2 / 2$ & $2 / 2$ & $2 / 2$ & $2 / 2$ & $2 / 2$ & $0 / 2$ & $0 / 2$ \\
\hline
\end{tabular}




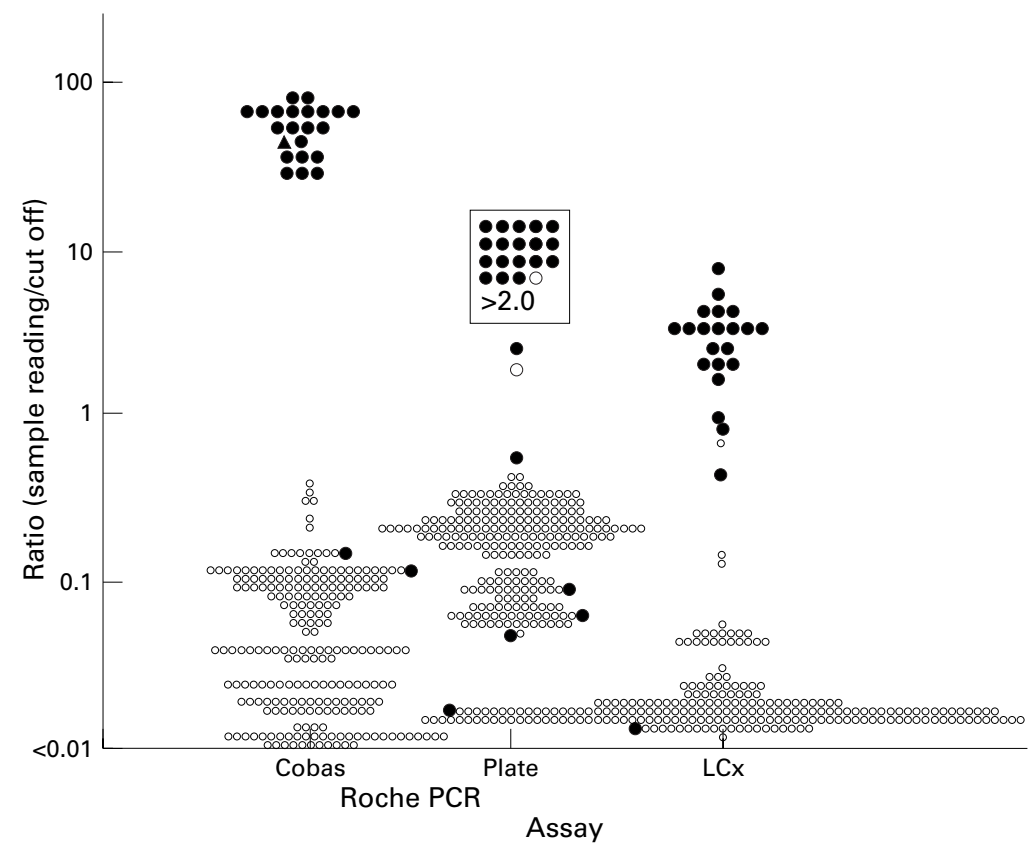

Figure 1 Comparison of sample reading/cut off ratios between commercial assays for 245 clinical specimens. Cut off $=1.00 ; \boldsymbol{O}=$ positive; $\bigcirc=$ negative; $\square=$ false positive; $\boldsymbol{\Lambda}=$ indeterminate. and plasmid numbers rather than EBs are used the true endpoints may be $1,1-2,2-3$, and 20 plasmid copies for the in house PCR, Roche Cobas Amplicor, Roche Amplicor plate kit (improved version), and LCR respectively.

A number of discrepant results were observed in this study. Initial in house PCR screening with plasmid primers gave 27 positive results, 23 of which were confirmed positive. Three unrepeatable positives with the plasmid primers were probably due to contamination during the PCR in spite of strict adherence to the rules for PCR performance. A sample from one asymptomatic patient who was repeatedly positive by the plasmid primers may have contained chlamydial DNA, but unfortunately no follow up sample was available to confirm this.

A number of workers have shown that patients can be PCR positive, even 4 weeks after treatment, ${ }^{13}$ while experimental work in monkeys has shown that PCR can detect chlamydia up to 5 weeks after samples become culture negative. ${ }^{14}$ In this study 10 specimens were from patients who had been diagnosed as positive and had returned for a check following treatment; two of these were positive; one by all assays and one by in house PCR and IF.

The Roche Cobas Amplicor gave positive results for 21 samples compared with 19 on the Roche plate kit. Since the clinical samples reported here were tested Roche have modified their plate kit. The modified version, used in the dilution series, appears to be closer in sensitivity to the Cobas.

The LCR gave no false positive results but failed to detect one sample positive by culture and two samples positive by in house PCR and IF. An additional sample which was in house PCR and Roche Cobas positive gave an LCR result just below the cut off. These false negatives could not be explained by an absence of the $7.5 \mathrm{~kb}$ cryptic plasmid in that the in house PCR primers used this target sequence for amplification. It is possible that a mutation could have prevented hybridisation of the probes to the target sequence, or LCR inhibitors may have been present. The presence of inhibitors for urines has been discussed previously for LCR in which prolonged storage and freeze thawing reduced the effects of these inhibitors. ${ }^{15}$ Since two of these missed positives were male urethral swabs the possibility of inhibitors being present cannot be ruled out. However, a more likely explanation was that the number of elementary bodies was very low in these specimens. The culture system used $500 \mu \mathrm{l}$ of specimen yet frequently detected extremely low numbers of inclusions in the culture. Smaller aliquots may not have contained any or very few elementary bodies even though before aliquoting, samples were vortexed for 1 minute to ensure homogeneous mixing. Low levels of plasmid DNA may be detected more efficiently by nested PCR, which increases theoretical sensitivities for single copies of target sequence. ${ }^{16}$

Previous work has suggested that the sensitivities of Roche PCR plate kit and Abbott LCR are equivalent and that the cut off level of both assays is $2-4$ elementary bodies, ${ }^{17}$ perhaps exe well beyond our budget. ever, it is also possible that the differences may be greater than shown. If the volumes used in each assay are allowed for in the calculation 
explaining their inability to detect a culture positive sample with a low level of elementary bodies detected by IF. In automating the system Roche have been able to increase sensitivity and specificity of their assay.

The in house PCR was $9 \%$ more sensitive than the Roche amplification system with a small loss in specificity (98\%). Processing time was reduced using Qiamp viral RNA extraction columns with a vacuum manifold. This increased sample throughput considerably when compared with conventional phenol/chloroform extraction methods. The extraction method allows the processing of 48 samples within an hour and positive results could be confirmed within 48 hours. If the in house PCR was used for routine diagnosis, all plasmid positives would be confirmed with MOMP and/or IF before reporting to the clinician. The plasmid primers were more sensitive than the MOMP primers as shown by the two plasmid/IF positive but MOMP primer negative samples. One sample was repeatedly plasmid positive but MOMP and IF negative which might indicate that some samples may be unconfirmable; these would have to be reported as indeterminate and a repeat sample supplied.

The semiautomated commercial assays had distinct advantages over the in house PCR from the technician's point of view in that the preparation step before amplification was much simpler and the hands on time thereafter was much less. When large numbers of samples have to be processed every day the risk of operator error increases and this risk was greatest with the in house assay. A speedier result was the other main advantage of the commercial assays. The advantages of the in house assay in addition to greater sensitivity were cost and the fact that the result would be confirmed before reporting. However the royalty payment to Roche would decrease cost savings advantages.

The demonstrated lower sensitivity of culture means that it can no longer be regarded as the gold standard for $C$ trachomatis. In addition, variations in sensitivity from centre to centre, the need for well taken specimens transported rapidly in cold conditions, and its unsatisfactory detection rate in urines all make it more fallible than molecular methods. The additional positives detected by in house PCR increased the detection rate from $8 \%$ (as previ- ously reported in this laboratory) ${ }^{12}$ to $9.5 \%$. These increased detection rates are also seen with the commercial assays. An expanded gold standard should include the use of nucleic acid amplification technologies, either in house PCRs or commercially available tests, because of their higher sensitivities.

We would like to thank Azra Sharif-Qayyum for expert technical assistance in the routine culture, Roche Diagnostics for the supply of Amplicor PCR kits and Abbott for the use of the LCx system.

Contributors: RMS and CP did the laboratory bench work. PS and SS were responsible for the design and interpretation and for obtaining clinical details.

Further details of the materials and methods used may be Further details of the materials
obtained from the journal offices.

1 Ripa K T, Mardh PA. Cultivation of Chlamydia trachomatis in cycloheximide treated McCoy cells. 7 Clin Microbiol 1977;6:328-31.

2 Mahony J, Cherensky M. Effect of swab type and storage temperature on the isolation of Chlamydia trachomatis from clinical specimens. F Clin Microbiol 1985;22:865-7.

3 Mardh PA, Zeeberg B. The toxic effect of sampling swabs and transport test tubes on the formation of intracytoplasand transport test tubes on the formation of intracytoplasmic inclusions of

4 Birkenmeyer L, Mushahwar IK. DNA probe amplification methods. F Virol Methods 1991;35:117-26.

5 Lee HL, Cherensky MA, Schachter J. Diagnosis of Chlamydia trachomatis genitourinary infection in women by ligase chain reaction assay of urine. Lancet 1995;345:213-16

6 Schachter J, Stamm WE, Quinn TC, et al. Ligase chain reaction to detect Chlamydia trachomatis infection of the cervix. F Clin Microbiol 1994;32:2540-3.

7 Palmer L, Falkow S. A common plasmid of Chlamydia trachomatis. Plasmid 1986;16:52-62.

8 Evans RT, Woodland RM. Detection of chlamydiae by isolation and direct examination. Br Med Bull 1983;39:181-6.

9 Thomas BJ, Evans RT, Hutchinson GR, et al. Early detection of Chlamydia inclusions combining the use of detection of Chlamydia inclusions combining the use of
cycloheximide treated McCoy cells and immunofluorescycloheximide treated McCoy cells and imm
cence staining. 7 Clin Microbiol 1977;6:285-92.

10 Carty MA, Borrego MJ, Cardoso J, et al. Comparison of Amplicor Chlamydia trachomatis test and cell culture for the detection of urogenital chlamydial infections. Genitourin Med 1995;71:247-50

11 Mahony JB, Linstra KE, Sellors JW, et al. Comparison of plasmid and chromosome based-polymerase chain reaction assays for detecting Chlamydia trachomatis nucleic acids. $f$ Clin Microbiol 1993;31:1753-8.

12 Smith IW, Morrison CL, Patrizio C, et al. Use of a commercial PCR kit for detecting Chlamydia trachomatis. $\mathcal{F}$ Clin Pathol 1993; 46:822-5.

13 Holland SM, Hudson AP, Bobo L. Demonstration of chlamydial RNA during culture negative state. Infect Immunol 1992;60:2040-7.

14 Bobo L, Munoz B, Viscidi R, et al. Diagnosis of Chlamydia trachomatis eye infection in Tanzania by polymerase chain reaction/enzyme immunoassay. Lancet 1991;338:847-50.

15 Paul ID, Leece JL, Caul EO. Chlamydia trachomatis: review of its laboratory diagnosis and preliminary evaluation of a new DNA based assay. PHLS Micro Dig 1996;13: 223-6.

16 Simmonds P, Balfe P, Peutherer JF, et al. Human immunodeficiency virus-infected individuals contain provirus in small numbers of peripheral mononuclear cells and at low copy numbers. F Virol 1990;64:864-72.

17 Miyashita N, Matsumoto A, Niki Y, et al. Evaluation of the sensitivity and specificity of the ligase chain reaction kit for the detection of Chlamydia trachomatis. 7 Clin Pathol 1996;46:515-17. 